\title{
The Effects of Bench Press Variations in Competitive Athletes on Muscle Activity and Performance
}

\author{
by \\ Atle Hole Saeterbakken ${ }^{1}$, Dag-André Mo ${ }^{1}$, Suzanne Scott², Vidar Andersen ${ }^{1}$
}

The aim of the study was to compare the EMG activity performing 6RM competition style bench press (flat bench-wide grip) with 1) medium and narrow grip widths on a flat bench and 1) inclined and declined bench positions with a wide grip. Twelve bench press athletes competing at national and international level participated in the study. EMG activity was measured in the pectoralis major, anterior and posterior deltoid, biceps brachii, triceps brachii and latissimus dorsi. Non-significant differences in activation were observed between the three bench positions with the exception of 58.5-62.6\% lower triceps brachii activation, but 48.3-68.7\% greater biceps brachii activation in the inclined bench compared with the flat and declined bench position. Comparing the three grip widths, non-significant differences in activations were observed, with the exception of 25.9-30.5\% lower EMG activity in the biceps brachii using a narrow grip, compared to the medium and wide grip conditions. The 6-RM loads were $5.8-11.1 \%$ greater using a medium and wide grip compared to narrow grip width and 18.5-21.5\% lower in the inclined bench position compared with flat and declined. Comparing the EMG activity during the competition bench press style with either the inclined and declined bench position (wide grip) or using a narrow and medium grip (flat bench), only resulted in different EMG activity in the biceps- and triceps brachii. The 6RM loads varied with each bench press variation and we recommend the use of a wide grip on a flat bench during high load hypertrophy training to bench press athletes.

Key words: Resistance, training, strength, performance.

\section{Introduction}

Performance during the bench press exercise is measured as the maximum load that can be lowered to the chest and raised ('pressed') above the body to full elbow extension (Baechle and Earle, 2008). Competitive bench press athletes use numerous variations in the bench press to isolate and train shoulder girdle muscles, which contribute significantly to performance. Understanding the effect of manipulating variables has the potential to improve program design and increase the specificity of hypertrophy training protocols (Bird et al., 2005; Kraemer and Ratamess, 2004; Stastny et al., 2017).

Previous studies have examined the kinematics of the bench press action (Madsen and

\begin{abstract}
McLaughlin, 1984), the effect of different chest press exercises (Welsch et al., 2005), unstable surfaces (Anderson and Behm, 2004), the impact of fatigue (Golas et al., 2016; van den Tillaar and Saeterbakken, 2013), as well as successful and unsuccessful attempts (van den Tillaar and Ettema, 2009), and different approaches (concentric vs counter movement (Tillaar and Ettema, 2013); isometric vs dynamic (Tillaar et al., 2012)) in bench press and chest press exercises with maximal and/or submaximal loads (Saeterbakken et al., 2011). However, there is limited evidence on the effects of different grip widths and inclinations of the bench on muscle activation patterns during execution. For example,
\end{abstract}

1 - Department of Teacher Education and Sports, Sogn and Fjordane University College, Sogndal, Norway.
2 - The Scott Studio, Pithers Yard, High St, Castle Cary, Somerset, BA7 7AN, UK. 
athletes commonly adjust grip width with the aim of increasing the specificity of muscle activation. However, evidence supporting this as an effective strategy is not conclusive. Increasing the grip width to $200 \%$ of the biacromial distance resulted in greater pectoralis major (PM) (Barnett et al., 1995; Clemons and Aaron, 1997; Lehman, 2005), but lower anterior deltoid (AD) (Barnett et al., 1995) and triceps brachii (TB) activation compared with biacromial grip width (Barnett et al., 1995; Lehman, 2005; Maszczyk et al., 2016). In addition, increased biceps brachii (BB) activation has been demonstrated for a wide compared to a narrow grip (Lehman, 2005).

Varying the bench position (inclined and declined) has been shown to bias activation in either the sternal or clavicular portion of the PM (Barnett et al., 1995; Glass and Armstrong, 1997; Lauver et al., 2016; Trebs et al., 2010). Increased activation of the clavicular part of the PM has been demonstrated on an inclined bench (Trebs et al., 2010), with reduced activation of sternocostal fibers of the PM compared with the flat bench position (Barnett et al., 1995; Trebs et al., 2010). Barnett et al. (2015) demonstrated a similar pattern of increased activation of the sternocostal PM, but only an increase of the clavicular PM at the inclined compared with declined bench position. In contrast, Glass and Armstrong (1997) observed a similar increase in clavicular activation, with a greater increase in sternocostal PM activation in the declined position compared with an inclined bench. The inconclusive evidence from aforementioned studies could be the result of different inclined and declined bench positions (greater position of inclination compared with the position of decline) or that only positions of inclination were assessed (Barnett et al., 1995; Glass and Armstrong, 1997; Lauver et al., 2016; Trebs et al., 2010). Previous bench press studies were also limited by having examined recreational or strength-trained participants rather than competitive bench press athletes, testing muscle activation patterns at non-fatiguing, submaximal loads, and applying the criterion of absolute rather than relative intensity when comparing different variations of the bench press action (Barnett et al., 1995; Clemons and Aaron, 1997; Glass and Armstrong, 1997; Lauver et al., 2016; Trebs et al., 2010).

The neuromuscular effects of applying systematic variations in grip widths and bench positions at maximal training intensity have not been examined to date in elite bench press athletes. Therefore the aim of the study was to compare the electromyographic (EMG) activity in clavicular and sternocostal parts of the pectoralis major, triceps brachii, biceps brachii, anterior deltoid, posterior deltoid and latissimus dorsi during the competition style bench press with 1) a medium and narrow grip on a flat bench, and 2) a wide grip on an inclined and declined bench under a 6RM load.

\section{Material and Methods}

\section{Participants}

Twelve bench press athletes competing at national and international level (mean age $34.3 \pm$ 14.1 years, body mass $97.6 \pm 18.3 \mathrm{~kg}$, stature $1.73 \pm$ $0.12 \mathrm{~m}$ ) participated in the study. All participants competed in the bench press, four athletes participated in all three competitive powerlifting exercises (bench press, squat and deadlift). For an overview of the participants' competition experience, personal records, national records and national/international participation in competitions see Table 1. Participants were excluded from the study if they had current musculoskeletal pain, illness or an injury that might affect production of maximal effort during testing. Participants were instructed to refrain from any additional resistance training 48 hours before testing.

\section{Ethics Statement}

The study was approved by the local ethical committee (2009/1735/REK Sør-Øst D) and conformed to standards of treatment of human participants in research as outlined in the $5^{\text {th }}$ Declaration of Helsinki. Participants were informed (both in writing and orally) about all testing and training procedures and gave written informed consent to participate prior to entering the study.

\section{Procedures}

A within-participants crossover design was used to compare 6-RM and EMG activity in the competition style bench press (flat bench wide grip) with: 1) narrow and medium grip width and 2) on a $+25^{\circ}$ inclined and $-25^{\circ}$ declined bench with a wide grip which was defined as the maximum grip width permitted in the bench 
press competition rules $(81 \mathrm{~cm}$ spacing between palms). Narrow grip width was set at participants' biacromial distance $(42.0 \pm 3.5 \mathrm{~cm})$ and medium grip width as $50 \%$ (wide - narrow grip; $61.5 \pm 3.5 \mathrm{~cm}$ ). Five sets of 6-RM presses were performed in randomized order. Before the experimental test, all participants performed two familiarization sessions in which individualized 6RM loads were identified for all five bench press conditions (familiarization 1: wide, medium and narrow grip; familiarization 2: wide grip on an inclined and declined bench). Between three and six sets with 5 minutes rest between each attempt were used to find the individualized loads, with 6-RM chosen as the typical training load recommended to gain strength in the training period in which the study was conducted (Ratemess et al., 2009). A minimum of six days separated the two familiarization sessions and the experimental test. Under experimental testing, all five sets of 6-RM were performed in one session.

Performance enhancing clothing and body strapping (e.g. bench shirt, elbow wraps) were not permitted during testing, but the participants were allowed to use lifting belts, wrist wraps and chalk to improve barbell grip. A $20 \mathrm{~kg}$ Olympic barbell (Leoko, Er Equipment, Denmark), an adjustable bench (Pivot 430 flexi bench, Sportsmaster, Norway) and a power rack (Pivot 480, Sportsmaster, Norway) were used for testing.

Before the familiarization sessions and experimental test, a standardized warm-up protocol typically used by the powerlifters was conducted. All warm-up sets were performed with the athletes' preferred grip width on a flat bench. The warm-up sets consisted of 20 repetitions with a barbell only (optional), 10 repetitions at $50 \%$, four repetitions at $70 \%$, two repetitions at $80 \%$ and one repetition at $90 \%$ of the 6-RM load. A three minute rest separated each warm-up set (Saeterbakken et al., 2011) with a five minute interval between each bench press variation.

The 6-RM lifts were not performed according to competition rules, which stipulate a deliberate stop phase in which the barbell rests on the chest, however, participants were instructed that the barbell had to touch the chest gently without any bouncing, before being lifted up to full elbow extension. Each repetition was performed at a self-selected speed and with no pauses between repetitions. Participants were instructed to maintain the head, shoulder blades and buttocks in contact with the bench in all variations. Two test leaders acted as spotters and assisted participants in the preload phases. Loads were decreased or increased if participants either failed to perform six repetitions, or indicated they could lift more.

\section{Recordings}

To identify the start and end of each repetition, a linear encoder was synchronized with the EMG recording system (Musclelab 4020e, Ergotest Technology A/S, Langesund, Norway). The linear encoder (at a sampling frequency of $100 \mathrm{~Hz}$ ) identified the vertical displacement (Figure 1a) in addition to the beginning, end and duration of each 6RM attempt. A goniometer (ss21 L, Biopac System Inc., USA) was attached to the fulcrum of the elbow, in line with the humerus and ulna (Figure 1b). The accuracy of the goniometer was $\pm 2^{\circ}$ measured over $90^{\circ}$, with repeatability of $< \pm 1^{\circ}$.

Before attempting the 6-RM lifts in the experimental test, participants' skin was shaved, abraded and washed with alcohol before placement of the self-adhesive electrode. Bipolar sliver-silver chloride electrodes $(11 \mathrm{~mm}$ contact diameter, $20 \mathrm{~mm}$ center-to-center distance) were placed over the center of the muscle belly along in the principal direction of muscle fibers of the pectoralis major (PM) (clavicular and sternocostal part), triceps brachii (TP), biceps brachii (BB), anterior deltoid (AD), posterior deltoid (PD) and latissimus dorsi (LD), using anatomical landmarks and according to the recommendations of SENIAM and similar studies (Hermens et al., 2000). The signal was amplified and filtered using a preamplifier located as near to the pickup point as possible. The preamplifier had a common mode rejection rate of $100 \mathrm{~dB}$. The raw EMG signals were band pass filtered (fourth-order Butterworth filter) with cut-off frequencies of 8$600 \mathrm{~Hz}$. The filtered EMG signals were root-mean square (RMS) converted using a hardware circuit network (frequency response $0-600 \mathrm{kHz}$, with an average constant of $100 \mathrm{~ms}$, total error $\pm 0.5 \%$ ). The RMS-converted signal was re-sampled at the rate of $100 \mathrm{~Hz}$ using a 16 bit analog-to-digital converter (AD637). The mean RMS value of all six repetitions in each condition was used for further 
analyses. Commercial software (Musclelab v8.13) was used to analyse the position data from the linear encoder, the elbow angle from the goniometer and the EMG data.

\section{Statistical analyses}

To assess differences in 6-RM, lifting time, vertical displacement of the barbell, elbow positions and EMG activity between each bench press variation: grip width (wide, medium and narrow) and inclination (flat, inclined, and declined), a repeated-measures analysis of variance (ANOVA) with Bonferroni post-hoc corrections were used. All calculations were performed using SPSS (version 20.0; SPSS, Inc., Chicago, IL, USA). Statistical significance was accepted at $p \leq 0.05$. All results are presented as means \pm SD and Cohen's d effect size (ES). An ES of 0.2 was considered small, 0.5 medium and 0.8 large (Cohen, 1988).

\section{Results}

\section{Bench positions}

Similar EMG activation in the PM (clavicular and sternocostal part), PD and LD in all three bench conditions (flat, inclined and declined) was observed ( $p=0.105-1.000$, Figure 2a, Table 2a). In the inclined bench condition,
EMG muscle activity in TB was $58.5 \%$ and $62.6 \%$ lower than in the flat and declined bench, respectively ( $p \leq 0.001-0.001$; $\mathrm{ES}=1.16-1.28)$. Similar TB activation was observed between the flat and declined bench $(p=1.000)$. In BB, $48.3 \%$ and $68.7 \%$ greater muscle activation was observed in the inclined bench condition compared with flat and declined, respectively $(p=0.003-0.005$, ES $=0.99-1.17)$, with no differences in EMG in BB observed between the flat and declined bench $(p=$ $0.401)$. In $A D$, similar EMG activity was observed between flat and the two inclined bench positions $(p=0.377-1.000)$, a $25.7 \%$ greater EMG activity was observed when performing in the inclined compared to the declined bench position $(p=$ 0.002; $\mathrm{ES}=0.50$ ).

\section{Grip widths}

No significant differences in any muscles were observed $(p=0.076-1.000)$ with the exception of the BB (Figure 2b, Table $2 b$ ). In the narrow grip, $\mathrm{BB}$ muscle activation was $30.5 \%$ and $25.9 \%$ lower than in the medium and wide grip, respectively $(p=0.003-0.040, \mathrm{ES}=0.25-0.33)$. Similar muscle activation was observed in the medium and wide grip $(p=1.000)$.
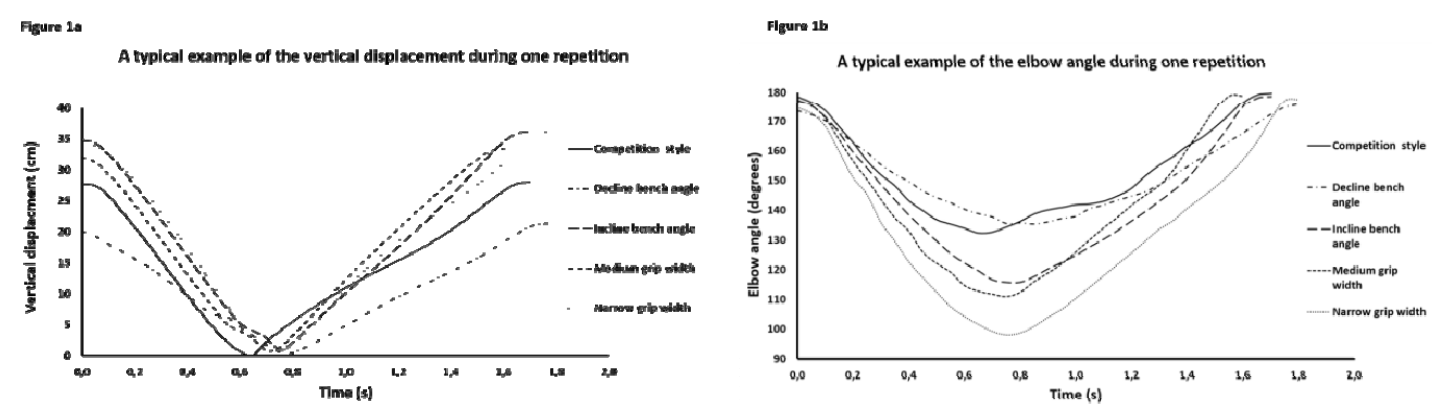

Figure 1a-b

The vertical displacement of the barbell (1a) and the elbow position (1b) performing the different bench press conditions. 


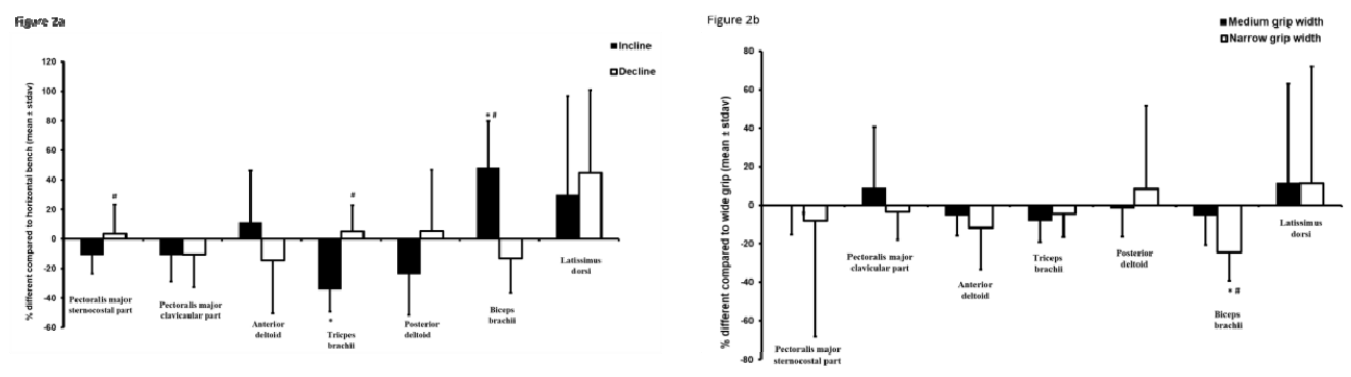

Figure 2a-b

The difference (\%) in muscle activation comparing inclined and declined (2a) and narrow and medium grip wide ( $2 b)$ with flat bench press.

\# significant difference between inclined and declined condition $(p<0.05)$.

* significant difference from all other bench positions $(p<0.05)$

\begin{tabular}{|c|c|c|c|c|c|c|}
\hline \multirow{15}{*}{$\#$} & \multicolumn{6}{|c|}{$\begin{array}{l}\text { Table } 1 \\
\text { An overview of the powerlifters bench press experience, personal best, } \\
\text { national records and national/international medals in championships. }\end{array}$} \\
\hline & $\begin{array}{l}\text { Weight } \\
\text { class }\end{array}$ & $\begin{array}{l}\text { Years competing } \\
\text { in bench press }\end{array}$ & $\begin{array}{l}\text { Personal best in } \\
\text { competition }(\mathrm{kg})\end{array}$ & $\begin{array}{l}\text { National } \\
\text { records }\end{array}$ & $\begin{array}{l}\text { Medals in national } \\
\text { championships }\end{array}$ & $\begin{array}{l}\text { Participation in } \\
\text { international } \\
\text { championships }\end{array}$ \\
\hline & $59 \mathrm{~kg}$ & 4 & 130 & 5 & 4 & \\
\hline & $-82.5 \mathrm{~kg}$ & 8 & 240 & & 3 & 3 \\
\hline & $-82.5 \mathrm{~kg}$ & 12 & 150 & & 7 & \\
\hline & $-93 \mathrm{~kg}$ & 9 & 220 & & & \\
\hline & $-93 \mathrm{~kg}$ & 4 & 217.5 & & 2 & \\
\hline & $-93 \mathrm{~kg}$ & 4 & 132.5 & & 1 & \\
\hline & $-93 \mathrm{~kg}$ & 4 & 245 & 9 & 5 & 1 \\
\hline & $-105 \mathrm{~kg}$ & 5 & 275 & 6 & 3 & 6 \\
\hline & $-105 \mathrm{~kg}$ & 4 & 215 & & & \\
\hline & $-105 \mathrm{~kg}$ & 3 & 200 & & & \\
\hline & $-120 \mathrm{~kg}$ & 4 & 220 & & 1 & \\
\hline & $+120 \mathrm{~kg}$ & 1 & 192.5 & & 1 & \\
\hline & \multicolumn{6}{|c|}{$\begin{array}{l}\text { significant difference between inclined and declined condition }(p<0.05) \text {. } \\
\quad * \text { significant difference compared to all other conditions }(p<0.05) \text {. }\end{array}$} \\
\hline
\end{tabular}


Table 2a

RMS values ( $\mathrm{mV}$ ) in flat, inclined and declined bench positions.

All values are presented as mean \pm standard deviation.

\begin{tabular}{cccc}
\hline Muscles & Wide & Medium & Narrow \\
\hline PM sternocostal part & $0.408 \pm 0.327$ & $0.390 \pm 0.293$ & $0.313 \pm 0.124$ \\
PM clavicular part & $0.505 \pm 0.317$ & $0.501 \pm 0.284$ & $0.457 \pm 0.244$ \\
Triceps brachii & $0.679 \pm 0.290$ & $0.616 \pm 0.228$ & $0.652 \pm 0.261$ \\
Anterior deltoid & $0.835 \pm 0.341$ & $0.775 \pm 0.276$ & $0.739 \pm 0.387$ \\
Posterior deltoid & $0.192 \pm 0.052$ & $0.190 \pm 0.064$ & $0.208 \pm 0.094$ \\
Biceps brachii & $0.227 \pm 0.167$ & $0.219 \pm 0.198$ & $0.174 \pm 0.152 *$ \\
Latissimus dorsi & $0.111 \pm 0.058$ & $0.111 \pm 0.058$ & $0.132 \pm 0.130$
\end{tabular}

* significantly different from all other bench positions $(p<0.05)$.

\begin{tabular}{|c|c|c|c|}
\hline \multicolumn{4}{|c|}{$\begin{array}{c}\text { Table } \mathbf{2 b} \\
\text { RMS values }(m V) \text { of the wide, medium and narrow bench grip width. } \\
\text { All values are presented as mean } \pm \text { standard derivation. }\end{array}$} \\
\hline Muscles & Horizontal & Inclined & Declined \\
\hline PM sternocostal part & $0.344 \pm 0.341$ & $0.287 \pm 0.234$ & $0.348 \pm 0.370$ \\
\hline PM clavicular part & $0.492 \pm 0.293$ & $0.425 \pm 0.218$ & $0.444 \pm 0.322$ \\
\hline Triceps brachii & $0.767 \pm 0.302$ & $0.484 \pm 0.164 *$ & $0.787 \pm 0.291$ \\
\hline Deltoideus anterior & $0.705 \pm 0.297$ & $0.747 \pm 0.294$ & $0.594 \pm 0.306$ \# \\
\hline Deltoideus posterior & $0.262 \pm 0.071$ & $0.195 \pm 0.089$ & $0.275 \pm 0.134$ \\
\hline Biceps brachii & $0.174 \pm 0.062$ & $0.258 \pm 0.103 *$ & $0.152 \pm 0.076$ \\
\hline Latissimus dorsi & $0.100 \pm 0.054$ & $0.125 \pm 0.084$ & $0.145 \pm 0.105$ \\
\hline
\end{tabular}

\section{6-RM loads}

The 6-RM load in the inclined bench condition $(109.2 \pm 11.1 \mathrm{~kg})$ was $21.5 \%$ lower than in the flat condition $(132.7 \pm 17.1 \mathrm{~kg})$ and $18.5 \%$ lower than in the declined condition (129.4 \pm 13.7 $\mathrm{kg}, p \leq 0.001$, ES = 1.62-1.63). No difference was observed between the flat and declined position $(p$ $=0.212$ ). Comparing the $6-\mathrm{RM}$ loads in the different grip widths, $5.8 \%$ and $11.1 \%$ greater 6 RM loads were achieved in the wide grip condition $(132.7 \pm 17.0 \mathrm{~kg})$ compared with the medium $(125.4 \pm 17.4 \mathrm{~kg})$ and narrow grip $(119.2 \pm$
$16.6 \mathrm{~kg})$, respectively $(p \leq 0.001$, ES $=0.42-0.80)$. Furthermore, a greater 6-RM load was achieved using a medium grip compared with a narrow grip $(p=0.016$, ES $=0.36)$.

Comparing the elbow position at the lowest bar position, a greater position was measured in the declined compared with the inclined bench position $(p=0.001)$. No differences in the elbow position were found between the flat and declined or inclined bench positions $(p=$ 0.070-0.791). Comparing the grip widths, the wide grip induced a greater elbow position than the 
narrow and medium grip condition $(p=0.025-$ 0.047). No differences in the elbow position were observed between the narrow and medium grip ( $p$ $=0.369$ ).

Comparing the vertical displacement of the barbell, there was no difference between the flat bench position and the two inclinations $(p=$ 0.180-0.237), but a lower displacement was observed when performing using the declined compared to the inclined bench position $(p=$ $0.001)$. For the grip widths, a greater displacement was observed performing a narrow grip compared with a wide grip $(p=0.025)$. No differences were observed between wide and medium grip width $(p=0.483)$ or between medium and narrow grip width $(p=0.124)$.

Similar lifting times were registered for the different bench press variations (bench inclination; $p=0.219-1.000$ and grip widths; $p=$ $0.357-1.000)$.

\section{Discussion}

The main finding of this study is that performing a 6-RM bench press on an inclined bench resulted in lower triceps brachii activation, but higher biceps brachii activation compared with the flat and declined bench position. Comparing the three grip widths, similar muscle activation was observed, with the exception of reduced EMG activity in the biceps brachii using the narrow grip, compared to the medium and wide grip conditions. While 6-RM loads were found to increase as the grip position widened, lower 6-RM loads were achieved in the inclined bench position compared with the flat and declined bench positions, which may be of relevance in designing effective hypertrophy protocols.

Similar levels of activity in the PM (sternocostal and clavicular parts) were observed in the flat bench compared with the inclined and declined bench positions. Importantly, all participants were bench press athletes competing at national and international level and all participants used different bench press variations in their weekly training. This may have resulted in similar PM EMG activity in all three bench inclinations as the participants' training enabled them to adjust their shoulder joint and trunk alignment to maximize the contributions of the PM during the different bench press positions. A one year study that observed elite weight-lifters lifting strategies found only slight differences in muscle activity on repeated EMG measurement (Hakkinen et al., 1987) suggesting elite lifters exhibit repeatable muscle activation patterns on different tasks. It is possible that athletes in this study may have adjusted their lifting action to preserve consistent muscle activation, although we did not use 3D motion analysis to examine this aspect.

Previous comparable studies have demonstrated inconclusive EMG results in the sternocostal and clavicular part of the PM (Barnett et al., 1995; Glass and Armstrong, 1997; Trebs et al., 2010). In line with the present study, similar EMG activity in the clavicular part of the PM between the flat bench and the inclined and declined bench positions has been shown (Barnett et al., 1995). This latter study, in contrast to the present one, demonstrated greater activation in the clavicular part of the PM when comparing inclined and declined bench press positions, although only six recreationally trained male subjects participated, and unlike our study, a $+40^{\circ}$ inclined and $-18^{\circ}$ declined bench was used, which may explain the differences in our findings.

Elsewhere the effect of different inclined bench press positions has been examined with findings of similar activation in the clavicular part of the PM, but greater activation of the sternocostal part of the PM using a $28^{\circ}$ incline bench position compared with the flat BP (Trebs et al., 2010). This study used a Smith machine which imposes a fixed, one dimensional vertical lifting pathway, compared with the weighted barbell used in the present study. It is possible that constraining the motion pathway during the bench press action may result in stereotypical patterns of muscle activation compared with subtle adjustments in the glenohumeral joint and elbow position permitted by a weighted barbell in an experienced lifter.

The inclined bench position resulted in lower EMG activity in the TB, but greater $\mathrm{BB}$ activity, compared to both flat and declined bench positions. The biceps brachii is a bipartite muscle, with a long head originating in the supraglenoid tubercle of the scapula and a short head which originates close to the pectoralis minor on the coracoid process of the scapula, and as well as acting on the scapula also is a powerful supinator 
of the forearm. It is therefore possible to speculate from its anatomy that in the inclined position increased activity recorded in BB may reflect its action on the scapula, contracting isometrically to control the scapula in an inclined (as opposed to flatly aligned or declined) position and provide anchorage during the dynamic phase of the press. Alternatively, or additionally, greater BB activation may result from its action on the forearm and indirectly the wrist, resisting a greater pronatory moment caused by a greater internal rotation moment proximally at the glenohumeral joint, as the increased EMG activity recorded in pectoralis supports.

Performing the inclined bench press, the hit point of the barbell was more superior to the sternum than the other bench positions. This resulted in a lower elbow position (Figure 1b) compared with the flat and declined bench positions. In addition, the motion pathway of the barbell was more vertical with an inclined bench position than a reversed $C$ observed in the flat bench press (van den Tillaar and Ettema, 2010). Finally, greater activation in the BB may have resulted in reduced TB activation (Saeterbakken et al., 2011). All these factors taken together may have contributed to the BB and TB results. Similar TB results to the present study have been already reported (Barnett et al., 1995) but, unfortunately, Barnett et al. (1995) did not include an EMG measurement of the BB. To the authors' knowledge, no previous studies have included measurement of the EMG activity in the BB when examining the effect of different bench positions making our results difficult to compare with previous studies.

With regard to the effect of the bench position, this study found similar activation between the flat and the other two bench positions examined in the $\mathrm{AD}$, which is in contrast to Barnett et al. (1995) and Trebs et al. (2010) who demonstrated increased activation with increasing inclination. From the anatomical position of the shoulder joint, the incline bench position may result in a shoulder flexion rather than a flat abduction, which may benefit a greater $\mathrm{AD}$ activation. However, the unique selection of participants (competition level bench press athletes) may have resulted in similar neural activation of the flat bench press compared with the inclined and declined bench positions.
Despite a $45 \%$ difference in LD muscle activation between the incline and decline positions, no statistical difference was observed for any of the bench press positions, which may be due to low statistical power. A decline bench position would suggest a greater activation of the LD to avoid a flat barbell pathway, as demonstrated elsewhere (Barnett et al., 1995).

Lower 6-RM loads were observed on an inclined bench press when compared to the flat and decline bench press positions, which is supported by previous studies (Glass and Armstrong, 1997; Trebs et al., 2010). The difference in vertical displacement and training specificity explains the different 6-RM loads observed (Glass and Armstrong, 1997; Trebs et al., 2010).

Biceps brachii was the only muscle which demonstrated lower activation using the narrow grip than the medium and wide grip width. Using a narrow grip, the elbows tended to adduct towards the trunk, thereby increasing the sagittal flexion and reducing the transverse flexion of the shoulder joint which could have contributed to the reduced biceps brachii contribution in results. In addition, a lower elbow position was observed using the narrow grip which may independently have affected the results. Finally, lower BB activation at a narrow grip may reflect inhibitory drive to this muscle in order to avoid cocontraction of the TB acting as a glenohumeral joint stabilizer, and affecting force generation and therefore successful execution of the elbow extension action. To our knowledge, no previous studies have examined the $\mathrm{BB}$ activation in the bench press with different grip width and these results are therefore difficult to compare with previous research. Surprisingly, no differences in TB activation were observed between the three grips. Previous studies have demonstrated greater activation using a narrow grip compared to a wide grip (Barnett et al., 1995; Lehman, 2005). However, both above mentioned studies used resistance-trained participants, but not competing bench press athletes. Participants in this study were bench press trained athletes and their specialized training background may have enabled positioning adjustment towards maximal prime mover activation, independently of grip widths.

In the present study, no significant 
differences were observed in prime movers (PM, $\mathrm{TB}$ and $\mathrm{AD}$ ) or the antagonists (PD and $\mathrm{LD}$ ) when comparing the wide, medium and narrow grip widths. The unique selection of participants who perform multiple bench press training sessions per week may explain these EMG results (Hakkinen et al., 1987). The present EMG results are partly supported by previous studies using participants who trained recreationally. For example, Lehman (2005) and Barnett et al. (1995) demonstrated similar PM activation (sternocostal part), examining grip widths comparable to the present study. Greater activation of the PM using a wide grip than when using a narrow grip was reported by Clemons and Aaron (1997). In contrast, Barnett et al. (1995) demonstrated greater activation in the clavicular part of the PM using a narrow grip compared to a wide grip. The contrasting results of the PM activation between the present and previous studies could be explained by the different bench press levels of the participants (Barnett et al., 1995; Clemons and Aaron, 1997; Lehman, 2005), using similar absolute loads and not similar relative intensity between the different grip widths (Clemons and Aaron, 1997; Lehman, 2005), performing nofatigue sets (Barnett et al., 1995), analyzing only the concentric lifting phase (Clemons and Aaron, 1997) and analyzing the mean prime muscles activation and not single muscles (Clemons and Aaron, 1997). To the authors' knowledge, no previous studies have examined the PD or LD during the bench press with different grip widths. The results are therefore difficult to compare with previous studies.

As expected, greater 6-RM loads were observed with different grip widths (wide > medium $>$ narrow). Training specificity and differences in vertical displacement are most likely the cause of the differences. The 6-RM results were as expected, but in contrast to Barnett et al. (1995), which is to our knowledge, the only study that have reported the 6-RM loads. Barnett et al. (1995) observed a 5\% non-significant difference between narrow and wide grip widths. However, Barnett et al. (1995) only examined six recreationally trained participants and low statistical power probably caused the nonsignificant difference.

As the aim of this study was to compare variations in the bench press at a consistent relative intensity (6RM) of exercise, normalized EMG data based on MVC were not obtained. A limitation therefore of this study is that it is difficult to compare results with those from studies in which normalization of EMG data to permit comparison between subjects and specific muscles has been followed. However, as the present study used dynamic contractions, in contrast to the isometric contractions favored by normalization procedures (Clarys, 2000; Farina, 2006) and given evidence of limitations of this approach for forceful contractions (Jobe et al., 1984), normalizing the RMS data in the present study is unlikely to have provided information relevant to the principal aim under investigation.

A further limitation of this study is that it was conducted in the preparation phase of the season, which is characterized by a higher volume of training but lower training intensity, making results difficult to compare with those obtained during the competitive phase of the season. Finally, EMG only gives an estimate of muscle activation. There will always be a possibility of crosstalk from neighboring muscles (Winter et al., 1994).

Training variation is an important principle in maximizing performance. In the present study, similar levels of activation were observed in chest, shoulder and back muscles compared with the competition bench press modality, however, small differences in arm flexor/extensor muscles were observed. Using variations in the bench press during training may reduce stress on shoulders, elbows and wrists, making it possible to increase the training volume without compromising neuromuscular activation. Importantly, we showed that reducing the absolute load by using an incline or narrow grip, resulted in a relatively similar neuromuscular activation, which may benefit athletes returning from rehabilitation or experiencing pain during heavy lifting,

In conclusion, comparing the competition bench press style (wide grip on a flat bench) resulted in similar muscle activation in chest and shoulder muscles compared with 1) an inclined and declined bench position and 2) a medium and narrow grip width. However, an inclined bench position demonstrated greater $\mathrm{BB}$, but lower $\mathrm{TB}$ activation than a flat and declined bench position. Generally, the 6-RM loads were greater with a 
wide grip and a flat bench position, compared

with the other variations of grip widths and bench positions.

\section{Acknowledgements}

We thank the athletes for their enthusiastic participation and acknowledge the students' participation in the data collection. The study was conducted without any founding from companies or manufacturers or outside organizations.

\section{References}

Anderson KG, Behm DG. Maintenance of EMG activity and loss of force output with instability. J Strength and Cond Res, 2004; 18: 637-640

Baechle TR, Earle RW. Essentials of strength training and conditioning. Champaign, IL: Human Kinetics; 2008

Barnett C, Kippers V, Turner P. Effects of variations of the bench press exercise on the EMG activity of five shoulder muslces. J Strength and Cond Res, 1995; 9: 222-227

Bird SP, Tarpenning KM, Marino FE. Designing resistance training programmes to enhance muscular fitness: a review of the acute programme variables. Sports Med, 2005; 35: 841-851

Clarys JP. Electromyography in sports and occupational settings: an update of its limits and possibilities. Ergonomics, 2000; 43(10): 1750-1762

Clemons JM, Aaron C. Effect of grip width on the myoelectric activity of the prime movers in bench press. J Strength and Cond Res, 1997; 11: 82-87

Cohen J. Statistical Power for the Behavioral Sciences. Lawrence Erlbaum Associates Inc. Hillsdale, New Jersy; 1988

Farina D. Interpretation of the surface electromyogram in dynamic contractions. Exerc Sport Sci Rev, 2006; 34(3): 121-127

Glass SC, Armstrong T. Electromyographical activity of the pectoralis muscle during incline and decline bench presses. J Strength and Cond Res, 1997; 11: 163-167

Golas A, Maszczyk A, Pietraszewski P, Stastny P, Tufano J, Zajac A. Effects of pre-exhaustion on the patterns of muscular activity in the flat bench press. J Strength and Cond Res, 2016, ahead of print

Hakkinen K, Komi PV, Alen M, Kauhanen H. EMG, muscle fibre and force production characteristics during a 1 year training period in elite weight-lifters. Eur J Appl Physiol Occup Physiol, 1987; 56: 419-427

Hermens HJ, Freriks B, Disselhorst-Klug C, Rau G. Development of recommendations for SEMG sensors and sensor placement procedures. J Electromyogr Kinesiol, 2000; 10: 361-374

Jobe W, Tibone E, Perry J, Moynes D. An EMG measurments during bicycle pedalling, in Biomechanics Symposium. Am J Sports Med, 1984; 11: 3-5

Kraemer WJ, Ratamess NA. Fundamentals of resistance training: progression and exercise prescription. Med Sci Sports Exerc, 2004; 36: 674-688

Lauver JD, Cayot TE, Scheuermann BW. Influence of bench position on upper extremity muscular activation during bench press exercise. Eur J Sport Sci, 2016; 16: 309-316

Lehman GJ. The influence of grip width and forearm pronation/supination on upper-body myoelectric activity during the flat bench press. J Strength Cond Res, 2005; 19: 587-591

Madsen N, McLaughlin T. Kinematic factors influencing performance and injury risk in the bench press exercise. Med Sci Sports Exerc, 1984; 16: 376-381

Maszczyk A, Gołaś A, Czuba A, Król H, Wilk M, Sńtastný P, Goodwin J, Kostrzewa M, Zając A. EMG analysis and modelling of flat bench press using artificial neural networks. S Afric J Res Sport, Phys Edu Rec, 2016; 38(1): 91-103 
Ratamess NA, Alvar BA, Evetoch TK, Housh TJ, Kibler WB, Kraemer WJ, Triplett NT. Progression models in resistance training for healthy adults. Med Sci Sports Exerc, 2009; 41: 687-708

Saeterbakken AH, van den Tillaar R, Fimland MS. A comparison of muscle activity and 1-RM strength of three chest-press exercises with different stability requirements. J Sports Sci, 2011; 29: 533-538

Stastny P, Golas A, Blazek D, Maszczyk A, Wilk M, Pietraszewski P, Petr M, Uhlir P, Zajac A. A systematic review of surface electromyography analyses of the bench press movement task. Plos One, 2017; 12: 2

Tillaar R, Ettema G. A comparison of muscle activity in concentric and counter movement maximum bench press. J Hum Kinet, 2013; 38: 63-71 Tillaar R, Saeterbakken AH, Ettema G. Is the occurrence of the sticking region the result of diminishing potentiation in bench press? J Sports Sci, 2012; 30: 591-599

Trebs AA, Brandenburg JP, Pitney WA. An electromyography analysis of 3 muscles surrounding the shoulder joint during the performance of a chest press exercise at several positions. J Strength Cond Res, 2010; 24: 1925-1930

van den Tillaar R, Ettema G. A comparison of successful and unsuccessful attempts in maximal bench pressing. Med Sci Sports Exerc, 2009; 41: 2056-2063

van den Tillaar R, Ettema G. The "sticking period" in a maximum bench press. J Sports Sci, 2010; 28: 529-535

van den Tillaar R, Saeterbakken AH. Fatigue effects upon sticking region and electromyography in a sixrepetition maximum bench press. J Sports Sci, 2013; 31: 1823-1830

Welsch EA, Bird M, Mayhew JL. Electromyographic activity of the pectoralis major and anterior deltoid muscles during three upper-body lifts. J Strength and Cond Res, 2005; 19: 449-452

Winter DA, Fuglevand AJ, Archer SE. Crosstalk in surface electromyography: Theoretical and practical estimates. J Electromyogr Kinesiol, 1994; 4: 15-26

\section{Corresponding author:}

\section{Atle Hole Saeterbakken}

Faculty of Teacher Education and Sport

Sogn og Fjordane University College, PB 133, 6851 Sogndal. Norway

Phone: 004757676044

Fax: 004757676333

E-mail: atle.saeterbakken@hisf.no 